\title{
ORSIm Detector: A Novel Object Detection Framework in Optical Remote Sensing Imagery Using Spatial-Frequency Channel Features
}

\author{
Xin Wu, Student Member, IEEE, Danfeng Hong ${ }^{\circledR}$, Student Member, IEEE, Jiaojiao Tian ${ }^{(\mathbb{},}$, \\ Jocelyn Chanussot $^{\circledR}$, Fellow, IEEE, Wei Li ${ }^{\circledR}$, Senior Member, IEEE, \\ and Ran Tao ${ }^{\circledR}$, Senior Member, IEEE
}

\begin{abstract}
With the rapid development of spaceborne imaging techniques, object detection in optical remote sensing imagery has drawn much attention in recent decades. While many advanced works have been developed with powerful learning algorithms, the incomplete feature representation still cannot meet the demand for effectively and efficiently handling image deformations, particularly objective scaling and rotation. To this end, we propose a novel object detection framework, called Optical Remote Sensing Imagery detector (ORSIm detector), integrating diverse channel features extraction, feature learning, fast image pyramid matching, and boosting strategy. An ORSIm detector adopts a novel spatial-frequency channel feature (SFCF) by jointly considering the rotation-invariant channel features constructed in the frequency domain and the original spatial channel features (e.g., color channel and gradient magnitude). Subsequently, we refine SFCF using learning-based strategy in order to obtain the high-level or semantically meaningful features. In the test phase, we achieve a fast and coarsely scaled channel computation by mathematically estimating a scaling factor in the image domain. Extensive experimental results conducted on the two different airborne data sets are performed to demonstrate the superiority and effectiveness in comparison with the previous state-of-the-art methods.
\end{abstract}

Manuscript received June 8, 2018; revised December 10, 2018 and January 14, 2019; accepted January 22, 2019. Date of publication February 27, 2019; date of current version June 24, 2019. This work was supported in part by the National Natural Science Foundation of China under Grant 91638201 , Grant 61571033, Grant 61331021, Grant 61421001, and Grant U1833203. (Corresponding author: Danfeng Hong.)

$\mathrm{X}$. Wu and R. Tao are with the School of Information and Electronics, Beijing Institute of Technology, Beijing 100081, China, and also with the Beijing Key Laboratory of Fractional Signals and Systems, Beijing Institute of Technology, Beijing 100081, China (e-mail: 040251522wuxin@163.com; rantao@bit.edu.cn)

D. Hong is with the Remote Sensing Technology Institute, German Aerospace Center, 82234 Weßling, Germany, and also with Signal Processing in Earth Observation, Technical University of Munich, 80333 Munich, Germany (e-mail: danfeng.hong@dlr.de).

J. Tian is with the Remote Sensing Technology Institute, German Aerospace Center, 82234 Weßling, Germany (e-mail: jiaojiao.tian@dlr.de).

J. Chanussot is with the GIPSA-Lab, Grenoble INP, CNRS, Universite Grenoble Alpes, F-38000 Grenoble, France, and also with the Faculty of Electrical and Computer Engineering, University of Iceland, 101 Reykjavik, Iceland (e-mail: jocelyn@hi.is).

$\mathrm{W}$. $\mathrm{Li}$ is with the College of Information Science and Technology, Beijing University of Chemical Technology, Beijing 100029, China (e-mail: liwei089@ieee.org).

Color versions of one or more of the figures in this paper are available online at http://ieeexplore.iee.org.

Digital Object Identifier 10.1109/TGRS.2019.2897139
Index Terms-Object detection, optical remote sensing imagery, rotation invariant (RI), spatial-frequency domains.

\section{INTRODUCTION}

$\mathbf{G}$ ENERALLY speaking, optical remote sensing imagery is collected from airborne or satellite sources in the range of $400 \sim 760 \mathrm{~nm}$. As a large amount of multispectral images or a very high-resolution red, green, and blue (RGB) images are freely available on a large scale, there is a growing interest in various applications, such as dimensionality reduction [1], [2], segmentation [3], [4], unmixing [5]-[8], data fusion [9]-[11], object detection and tracking [12]-[14], and classification or recognition [15]-[18]. In recent years, geospatial object detection has been paid much attention due to its importance in environmental monitoring, ecological protection, hazard responses, and so on. However, optical remote sensing imagery inevitably suffers from all kinds of deformations, e.g., variabilities in viewpoint, scaling, and direction, which results in performance degradation of the detection algorithm. In addition, objects in optical remote sensing imagery [19]-[23], such as cars and airplanes in Fig. 1, are generally small relative to the ground sampling distance with cluttered backgrounds. To overcome these challenges, object detection in the remote sensing community has been extensively studied since the 1980s.

Many benchmarks available in public, e.g., TAS aerial car detection data set ${ }^{1}$ and NWPU VHR-10 data set ${ }^{2}$ [24], [25], have contributed to spurring interest and progress in this area of remote sensing object detection. As the diversity of the database, many robust methods are born one after another in order to further improve the detection performances. Existing detection methods can be roughly categorized as follows [13]: template matching-based, knowledge-based, object-based, and machine learning-based methods and other variants. These approaches mostly fail to describe object features in a complete space with a densely set of scales.

\footnotetext{
${ }^{1}$ http://ai.stanford.edu/ gaheitz/Research/TAS/tas.v0.tgz

${ }^{2}$ The Vaihingen data were provided by the German Society for Photogrammetry, Remote Sensing and Geoinformation (DGPF).
} 


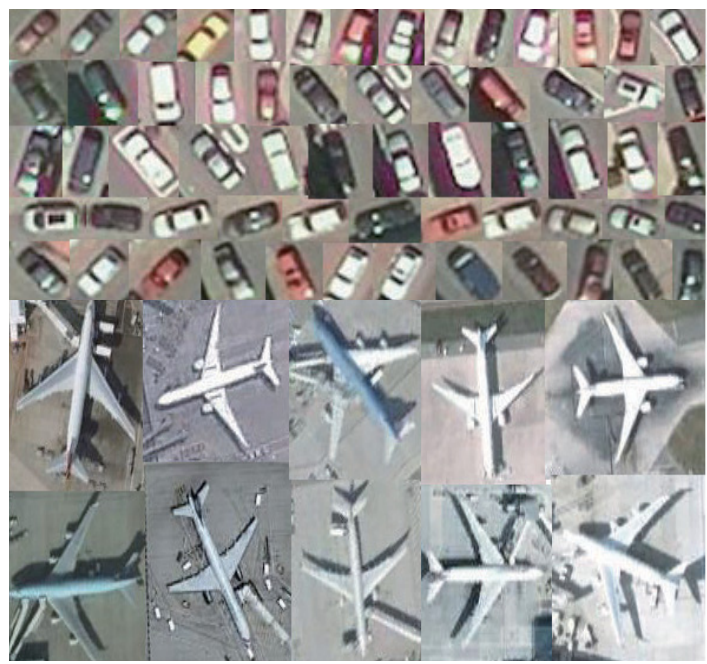

Fig. 1. Some seeds used in this paper for cars and airplanes object detection.

In our case, the so-called complete space should involve different properties robustly against various deformations, e.g., shift and rotation. Moreover, a good image descriptor should be able to capture substantial image patterns with a coarse image pyramid. We will detail them close to this paper and clarify the similarities and differences as well as pros and cons in Section II.

\section{A. Motivation and Objectives}

Object deformation (e.g., rotation and translation) in recognition or detection task is a common but still challenging problem. In particular, the remote sensing imagery is prone to have a more complex rotation behavior (see Fig. 1), due to its "bird perspective." Although the learning-based methods, such as deep neural networks (DNNs) and deep convolutional neural networks, have been proposed to learn the rotationinvariant (RI) features by manually augmenting the training set with different rotations, yet it is inevitably limited by the presetting rotation angles. This could be difficult to adaptively address the rotation problem of the fractional angle, thereby yielding a performance bottleneck. Another important factor that has a great effect on detection performance is the feature itself, which can be manually designed or extracted by DNN. However, such powerful learning approaches fail to provide a richer representation without the strong support of large-scale labeled training samples.

Consequently, we mainly make our efforts to artificially develop or optimize the features toward the more discriminative RI representations under the seminal object detection framework presented by Viola and Jones (VJ) [26], rather than the learning-based methods in this paper.

\section{B. Method Overview and Contributions}

To effectively address the aforementioned issues, the selfadaptive RI channel features [27] are first constructed in polar coordinates, which has been theoretically proven to well fit the rotation of any angles. Furthermore, the shift-invariant channel features in Cartesian coordinates [e.g., color and gradient magnitude (GM)] are also extracted for the channel extensions in order to fully explore the potential of the feature representation, yielding a joint spatial-frequency channel feature (SFCF). We then step toward feature learning or refine [e.g., subspace learning and aggregated channel features (ACF)] to further refine the representations. Such features are finally fed into a boosting classifier with a series of depth-3 decision trees.

For the geospatial object detection in remote sensing, we propose a variant of $\mathrm{VJ}$ object detection framework, called an Optical Remote Sensing Imagery detector (ORSIm detector). Unlike previous models in [27] and [28] that are sensitive to translations and rotations, the ORSIm detector is a more general and powerful framework robustly against various variabilities, particularly for remote sensing imagery. Additionally, a fast pyramid method is adopted to effectively investigate the multiscaled objects without sacrificing the detection performance. Fig. 2 outlines the basic framework of the ORSIm detector. The main highlights of this paper are threefold.

1) We propose a novel ORSIm detector by following the basic VJ framework by integrating SFCF, feature learning or refine, fast image pyramid estimation, and ensemble classifier learning (Adaboost [29]).

2) An SFCF is designed by simultaneously considering the invariance of rotation and shift in order to handle the complex object deformation behavior in remote sensing imagery.

3) An image pyramid generative model is simply but effectively embedded into the proposed framework by fast estimating a scaling factor in the image domain.

The remainder of this paper is organized as follows. Section II briefly reviews the previous work closely related to ours. Section III describes the proposed framework, including multiple domain feature exaction, feature stack, feature learning, training, and testing. The experimental results on two data sets are reported in Section IV. Section V concludes this paper and briefly discusses future work.

\section{RELATED WORK}

In this section, several advanced techniques in object detection are introduced with the applications to remote sensing imagery. We also emphatically clarify our superiority compared with three kinds of similar approaches partly associated with this paper.

\section{A. Channel Features}

Channel features refer to a collection of spatially discriminative features by linear or nonlinear transformations of the input image. Over the past decades, channel features extraction techniques have been received an increasing interest with successful applications in pedestrian detection [28], [30] and face detection [31]-[33]. Owing to their high representation ability, a variety of channel features have been widely used in geospatial object detection. Tuermer et al. [34] utilized the histogram of oriented gradients (HOG) [35] as orientation channel features for airborne vehicle detection in 


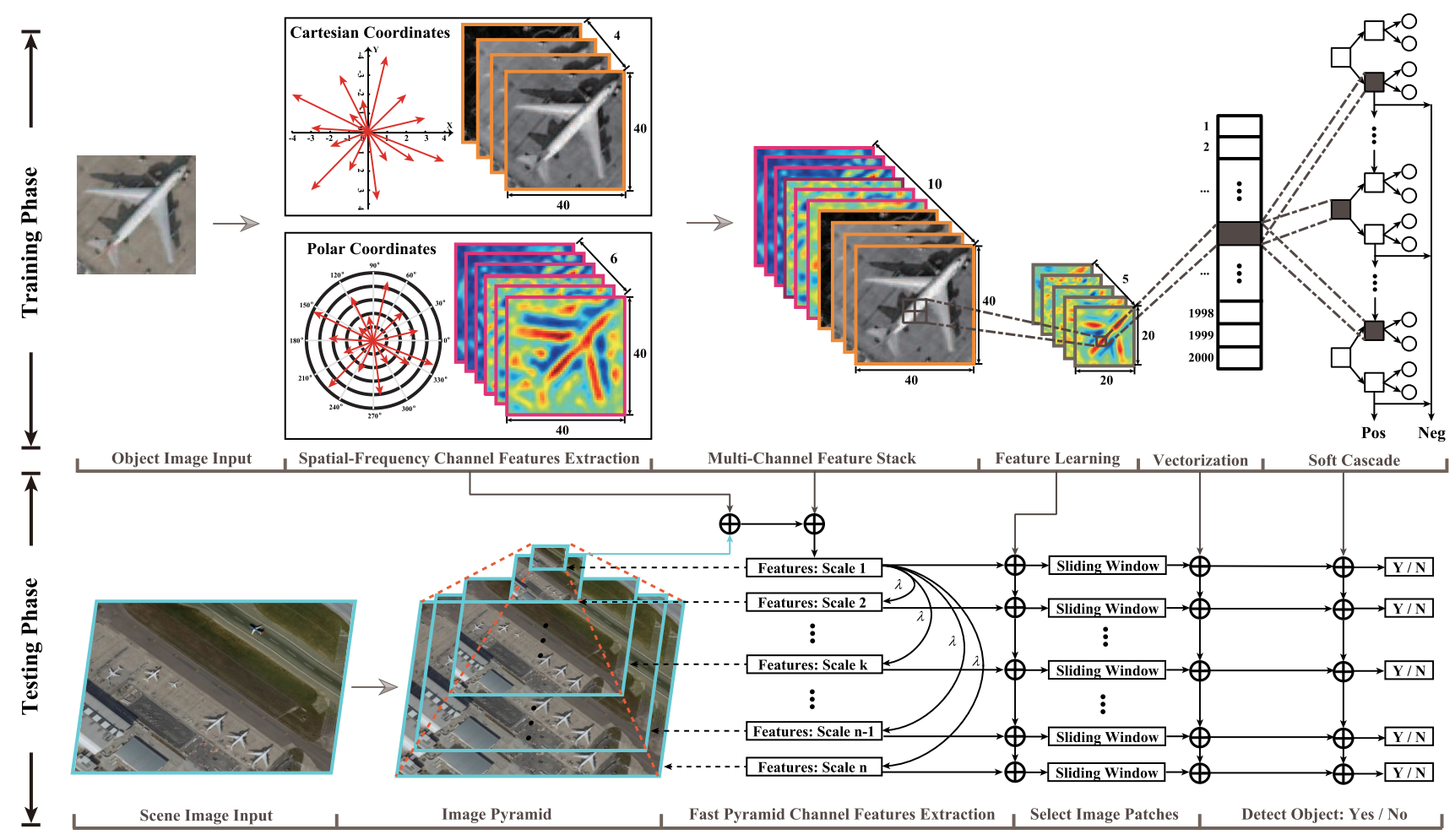

Fig. 2. Pipeline of the ORSIm detector, which is a concatenation of RI descriptor with low-sampled image pyramid and boosting tree model learned with respect to diverse tasks.

a dense urban scene. Unfortunately, using orientation features alone is prone to hinder the detection performance from further improving. Inspired by the ACF [28], Zhao et al. [36] extended the channel features by additionally considering the color channel features [e.g., gray scale, RGB, hue, saturation, and value (HSV), and luminance and chromaticity coordinates (LUV)] to detect aircrafts through remote sensing images. However, these methods usually fail to achieve desirable performances due to the sensitivity to object rotation. For that, although many tentative works have been proposed to model the object's rotation behavior [37], [38], yet the performance gain is still limited by the discrete spatial coordinate system.

With a theoretical guarantee, Liu et al. [27] proposed a Fourier HOG (FourierHOG) with a rigorous mathematical proof. It models the RI descriptor in a continuous frequency domain rather than in the discrete spatial domain using a Fourier-based convolutionally manipulated tensorvalued transformation function $D=P(r) e^{i m \varphi}$. This function transfers the tensor-valued vectorized features (e.g., HOG [39]) to a scalar-valued representation, so as to make the features invariant with a maximized information gain. In contrast with HOG-like approaches that discretely compute the features (or descriptors) in the locally estimated coordinates from pose normalization, FourierHOG uses a smooth continuous function for fitting the statistical features in a continuous coordinate, as shown in Fig. 3. Furthermore, such a strategy can also avoid artifacts in the gradient binning and pose sampling of the HOG descriptor.

Despite the superiority in representing rotation-invariance, FourierHOG ignores the importance of feature diversity. To this end, the proposed ORSIm extends the single-channel

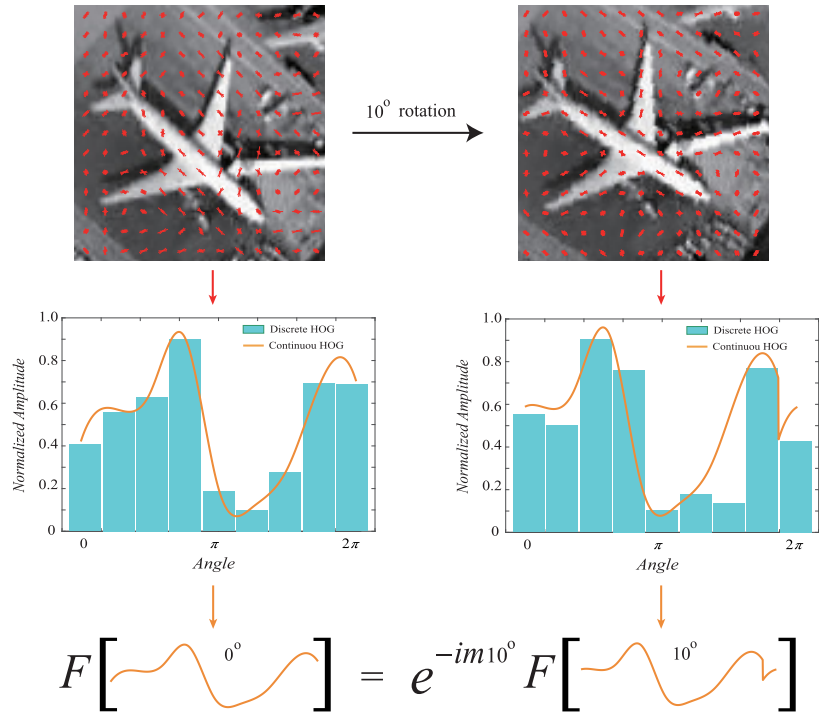

Fig. 3. Illustration of the discrete and continuous HOG distribution function of a cell $\left(13 \times 13\right.$ pixels). (Left) Reference HOG. (Right) $10^{\circ}$ rotated HOG. A property-rotated gap between the two discrete HOGs can be filled by shifting their corresponding continuous HOGs with $10^{\circ}$.

features toward spatial-frequency joint ones, thereby further enriching the representations. On the other hand, FourierHOG, in fact, simplifies a challenging problem of object detection to that of object recognition. More specifically, the task of detecting boundary box of the object is converted into that of recognizing the central pixel to be either object or nonobject, as shown in Fig. 4(a). 


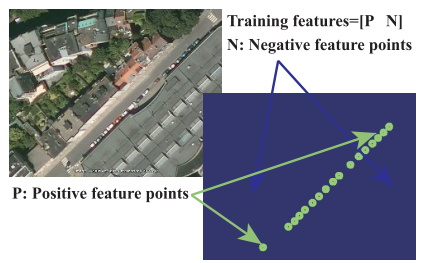

(a)

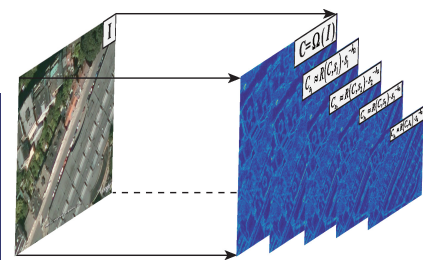

(b)
Fig. 4. Simple sketch map of training feature points extracting in [27] and feature channel scaling in [28]. (a) Training feature point. (b) Feature channel cubic.

\section{B. Feature Channel Scaling}

Image multiresolution decomposition is one of the essential techniques in high-level image analysis, such as object detection and tracking. The VJ framework is a seminal work for real-time object detection [26], [40], [41], which runs at $15 \mathrm{frames} / \mathrm{s}$ for an image of $384 \times 288$ pixels with a 700-MHz Intel Pentium III processor. Following this framework, HOG [35] yields a higher detection accuracy. Nevertheless, the two representative algorithms evenly sample scales in log-space and construct a feature pyramid for every scale. This is very time-consuming, and keeping the computational cost low is a significant challenge. Inspired by fractal statistics of natural images [42], Dollár et al. [28] proposed a fast pyramid generative model by only estimating a scale factor, basically achieving a pyramid feature extraction in parallel. The key technique used in the model can be summarized as a feature channel scaling, as shown in Fig. 4(b), the goal of which is to compute finely sampled feature pyramids at a fraction of the cost by means of the fractal statistics of images. Furthermore, the features are computed at octavespaced scale intervals in order to sufficiently approximate the features on a finely sampled pyramid. Therefore, these benefits make the model successfully applied to pedestrian detection at over 30 frames/s on an eight cores machine Inter Core i7-870 PC [43]. Similarly, it has been also proven to be effective in aircrafts detection of remote sensing images [36]. There is, however, an important assumption in the model, that is, the feature channels $\Omega$ are supposed to be any lowlevel shift-invariant in order to fit the operation of sliding windows, which makes the fast detection framework sensitive to angle variation or rotation-induced deformations. For this reason, Yang et. al. [31] attempted to relax the constraint by learning varied face properties from multiview images. The expensive cost of collecting multi-view remote sensing images still hinders Yang's algorithm from generalizing well. Congruously, either color channel features or FourierHOG is able to facilitate the use of the fast pyramid generative model, while their joint use (our SFCF) naturally does well.

\section{Boosting Decision Tree}

In the field of machine learning, the boosting methods have been widely used with great success for decades in various applications, e.g., object detection [12], [44], [45], face detection [26], and pose detection [46], [47]. Unlike other powerful classifiers [e.g., rotation-based support vector machine (SVM) [48], structured SVM [49], and rotation

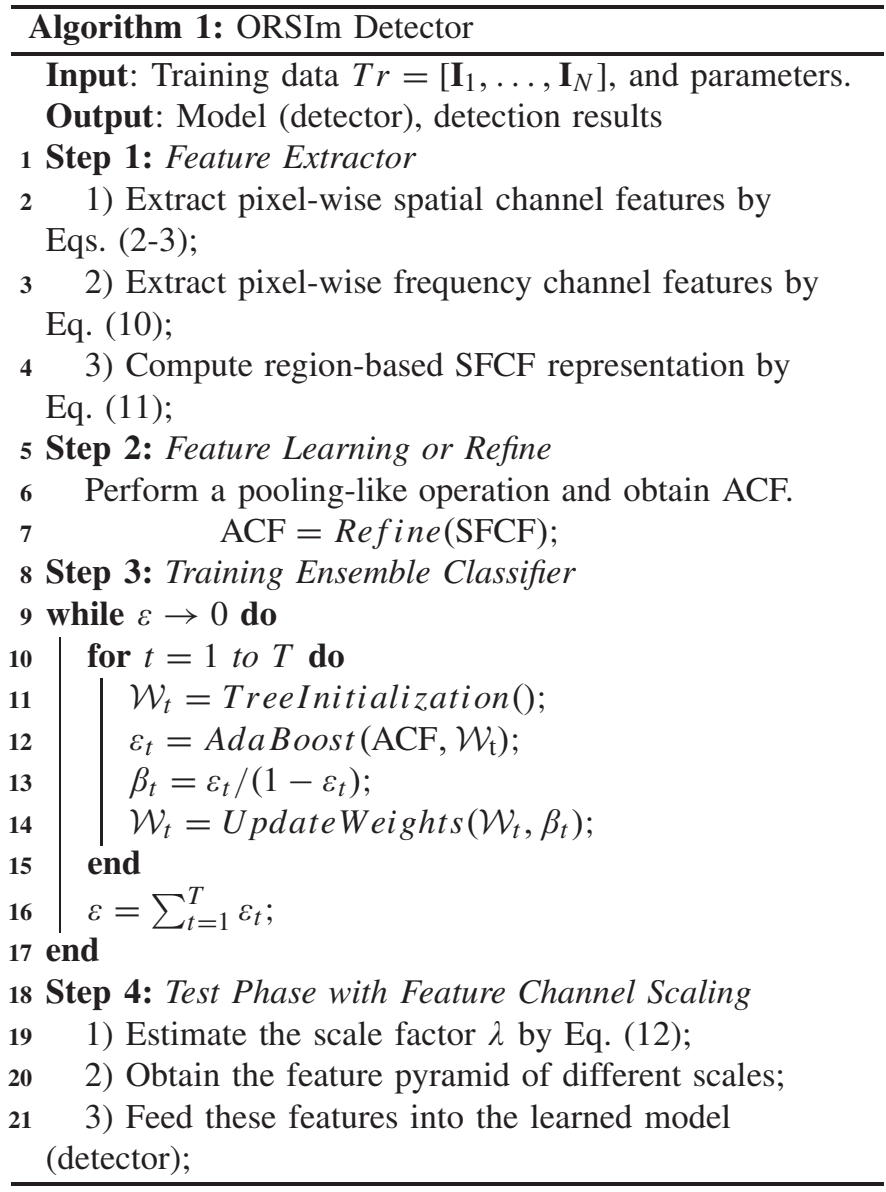

forest [50]], the boosting-based ones iteratively select weak learners from a pool of candidate weak classifiers to deal with hard examples from the previous round, which can be treated as an enhanced model integrating former results and greedily minimizing an exponential loss function. Each weak learner is able to make the sample reweighed; then, latter weak learners would more focus on those examples that are misclassified by former ones. Using this, a strong classifier can be learned with higher generalization ability and parameter adaptiveness.

The performance of boosting-based classifiers mainly relies on the discriminative ability of the feature and the number of weak classifiers. In Section III, we will introduce the proposed unified framework (ORSIm detector) in semantically meaningful feature extraction, feature stack, and learning as well as parameter selection of the boosting classifier.

\section{Methodology}

The proposed ORSIm detector starts with a feature extractor. At this stage, SFCFs are jointly extracted, including color and GM channels from the spatial-domain and RI features from the frequency domain. The features can be further refined by subspace learning or ACF, and then, they can be fed into boosting decision tree for a better training and detection. Algorithm 1 details the main procedures of the ORSIm detector.

\section{A. Spatial-Frequency Channel Features}

Commonly, the feature is limitedly represented in one single domain, and this motivates the joint extraction of more 
discriminative features from the spatial and frequency domains to enrich the feature diversity.

Given an RGB remote sensing imagery $\mathbf{I} \in \mathbb{R}^{L \times W \times 3}$ as the input, we denote $F_{\mathrm{SFCF}}$ as SFCF, mainly including the RGB channels, first-order GM channel, and RI channels, defined as

$$
F_{\mathrm{SFCF}}:=\{\underbrace{\Omega_{1}(\mathbf{I})}_{\mathcal{R} \mathcal{G B}}, \underbrace{\Omega_{2}(\mathbf{I})}_{\mathcal{G} \mathcal{M}}, \underbrace{\Omega_{3}(\mathbf{I})}_{\mathcal{R} \mathcal{I}}\}
$$

where $\left\{\Omega_{i}(\mathbf{I})\right\}_{i=1}^{3}$ stands for the different feature sets.

1) Pixelwise Spatial Channel Feature: In many tasks related to remote sensing, a color channel [51], i.e., RGB, shows a strong ability in identifying certain materials sensitive to the color (e.g., tree, grass, and soil), which can be denoted as

$$
\Omega_{1}(\mathbf{I})=\left[F_{R}, F_{G}, F_{B}\right]
$$

where $F$ represents the channel features. Moreover, the normalized GM for the RGB image can be regarded as another important spatial channel features, since it can not only sharpen the object edge, but also highlight the small mutations that could be visually ignored in the smooth areas of the image, which has shown its effectiveness in detecting aerial or spaceborne objects [34]. The resulting expression is

$$
\Omega_{2}(\mathbf{I})=F_{\mathrm{GM}} .
$$

2) Pixelwise Frequency Channel Feature: The objects in remote sensing images, more often than not, suffer from various complex deformations. It should be noted that object rotation is one of the major factors that sharply leads to the performance degradation. Compared with extracting features in Cartesian coordinates, rotation invariance has been proven to more effectively analyze in polar coordinates [27] where the feature can be separated as the angular information and radial basis $P(r)$, respectively. Let $\|\mathbf{d}\|$ and $\theta(\mathbf{d})$ be the magnitude and the phase of a complex number $\mathbf{d}=d x+d y i$, where $d x$ and $d y$ are the horizontal and vertical gradients of a pixel in Cartesian coordinates, respectively. Coincidentally, the Fourier basis $\psi_{k}(\varphi)=e^{i k \varphi}(k=0,1, \ldots, m)$ is an optimal choice for modeling the angular part $(\theta(\mathbf{d}))$, theoretically proven in [27], where $m$ stands for the Fourier order. The basis functions $\left[\psi_{0}, \psi_{1}, \ldots, \psi_{m}\right]$ form harmonics on a circle, called circular harmonics. In [27], the rotation behaviors $g(\bullet)$ in the Fourier domain can be modeled by a multiplication or convolution operator. More specifically, given two $k$ th order Fourier representations in polar coordinate $\left(f_{k_{p}}\right.$ and $f_{k_{q}}$ ), we have

$$
\begin{aligned}
g\left(f_{k_{p}} * f_{k_{q}}\right) & =e^{-i\left(k_{p}+k_{q}\right) \alpha_{g}}\left[f_{k_{p}} * f_{k_{q}}\right] \circ \mathbf{T}_{g} \\
g\left(f_{k_{p}} f_{k_{q}}\right) & =e^{-i\left(k_{p}+k_{q}\right) \alpha_{g}}\left[f_{k_{p}} f_{k_{q}}\right] \circ \mathbf{T}_{g}
\end{aligned}
$$

where $\mathbf{T}_{g}$ is a coordinate transform with a $\alpha_{g}$ relative rotation.

Given any one pixel $(\mathbf{p})$, its $k$ th order Fourier representations $\left(f_{k_{p}}\right)$ can be further deduced by

$$
f_{k_{p}}=\left\langle h, e^{i k_{p} \varphi}\right\rangle=\frac{1}{2 \pi} \int_{0}^{2 \pi} h(\varphi) e^{-i k_{p} \varphi}=\left\|\mathbf{d}_{k_{p}}\right\| e^{-i k_{p} \theta\left(\mathbf{d}_{k_{p}}\right)}
$$

where $h(\varphi)$ is the distribution function of current pixel, which can be modeled by an impulse function with integral $\left\|\mathbf{d}_{k_{p}}\right\|[27]: h(\varphi):=\left\|\mathbf{d}_{k_{p}}\right\| \delta\left(\varphi-\theta\left(\mathbf{d}_{k_{p}}\right)\right)$.

When (5) rotates by an angle $\alpha_{g}$, according to the rotation behavior $g \mathbf{d}:=\mathbf{R}_{g} \mathbf{d} \circ \mathbf{T}_{g}$ [27], we have

$$
\begin{aligned}
g f_{k_{p}} & =\left[\left\|\mathbf{R}_{g} \mathbf{d}_{k_{p}}\right\| e^{-i k_{p} \theta\left(\mathbf{R}_{g} \mathbf{d}_{k_{p}}\right)}\right] \circ \mathbf{T}_{g} \\
& =\left[\left\|\mathbf{d}_{k_{p}}\right\| e^{-i k_{p} \alpha_{g}} e^{-i k_{p} \theta\left(\mathbf{d}_{k_{p}}\right)}\right] \circ \mathbf{T}_{g} \\
& =e^{-i k_{p} \alpha_{g}}\left[f_{k_{p}} \circ \mathbf{T}_{g}\right] .
\end{aligned}
$$

In order to make the feature RI, namely $f_{k_{p}}=g f_{k_{p}}$, we can set a set of filters (convolution kernels) with the same rotation behavior, denoted as $f_{k_{q}}(k=0,1, \ldots, m)$. Using (4)-(6), this can be formulated as

$$
g\left(f_{k_{p}} * f_{k_{q}}\right)=e^{-i\left(k_{p}+k_{q}\right) \alpha_{g}}\left[f_{k_{p}} * f_{k_{q}}\right] \circ \mathbf{T}_{g}
$$

as long as satisfying $k_{p}+k_{q}=0$, we can get

$$
g\left(f_{k_{p}} * f_{k_{q}}\right)=\left[f_{k_{p}} * f_{k_{q}}\right] \circ \mathbf{T}_{g}
$$

thereby the convolutional features can be seen as the final RI representation.

Inspired by the above-mentioned theory derivation in terms of rotation invariance, we construct the RI features including the following three parts.

1) Using the Fourier transformation on the input remote sensing images, the magnitude channel image in the $k$ th Fourier order is naturally a kind of invariant feature, which is denoted as $F_{k_{p}}^{1}=\left\|\mathbf{d}_{k_{p}}\right\|(k=0,1, \ldots, m)$ in a pixelwise (p) form.

2) To make the representation absolutely RI, we get rid of rotation information from phase one by using (7) and (8). That is, we generate a series of Fourier basis with equal and opposite order and use them on the Fourier representations of $\mathbf{I}\left(f_{k_{p}}\right)$ by a multiplication or convolution operation, which can be formulated as $F_{k_{p}}^{2}=f_{k_{p}} * f_{k_{q}}$ and $k_{p}=-k_{q}$.

3) We also consider a relative RI feature representation by effectively utilizing the relative phase information [52]. Accordingly, this can be developed as a special RI feature by coupling the convolutional features of two neighboring kernel-radii (please refer to [27] for more details), which is formulated as $F_{k_{p}}^{3}=\left(f_{k_{p}} *\right.$ $\left.f_{k_{q}, r 1}\right) \overline{\left(f_{k_{p}} * f_{k_{q}, r 2}\right)} /\left\|\left(f_{k_{p}} * f_{k_{q}, r 1}\right) \overline{\left(f_{k_{p}} * f_{k_{q}, r 2}\right)}\right\|$ and $k_{p} \neq-k_{q} . r 1$ and $r 2$ stand for the different convolutional kernels.

Therefore, the pixel-based frequency channel feature can be written by

$$
\begin{aligned}
& \Omega_{3}(\mathbf{p}) \\
& \quad=\left[F_{0_{p}}^{1}, \ldots, F_{k_{p}}^{1}, \ldots, F_{0_{p}}^{2}, \ldots, F_{k_{p}}^{2}, \ldots, F_{0_{p}}^{3}, \ldots, F_{k_{p}}^{3}, \ldots\right] .
\end{aligned}
$$

Thus, we have the image-level representation by collecting all pixel-based features

$$
\Omega_{3}(\mathbf{I})=\left\{\Omega_{3}(\mathbf{p})\right\}_{p=1}^{L \times W} .
$$




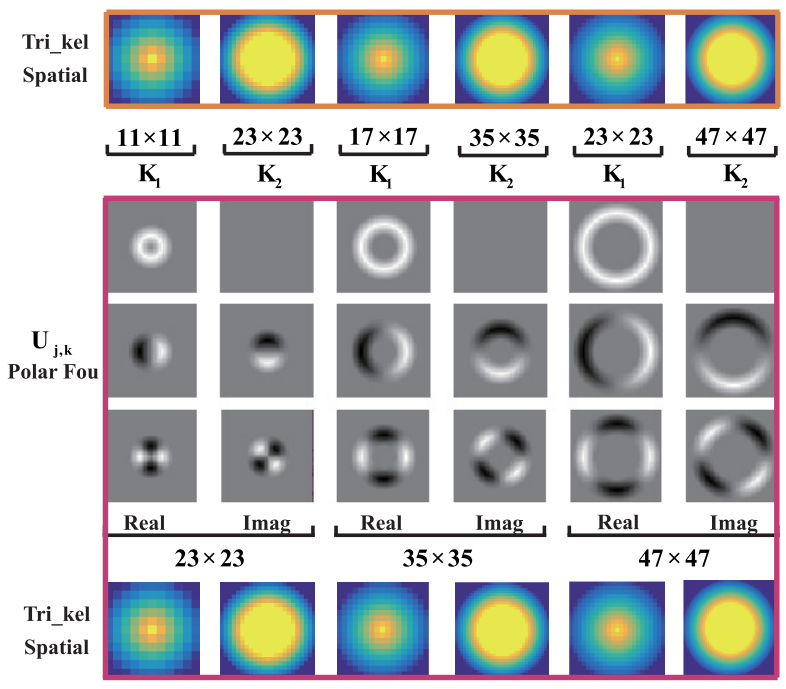

Fig. 5. Triangular convolution kernel of two domains. Spatial $\left[K_{1}\right.$ : the convolution kernel for the spatial aggregation. $K_{2}$ : the convolution kernel for the local normalization (based on gradient energy)]; $U_{j, k}$ : the basic function from triangular radial profile and a Fourier basis.

3) Region-Based Channel Feature Representation: Due to the low spatial resolution of remote sensing imagery, the detection performance is largely limited by the pixelwise features. To better capture the semantically contextual information, we group the pixelwise channel features into region-based ones with kernel functions of different sizes. As visualized in Fig. 5, we use the triangular convolution kernels, including isotropic triangles kernel and local normalization kernel, to extract the region-based channel features in both the spatial and frequency domains. Besides that, we additionally design a set of Fourierbased convolution kernels denoted as $U_{j, k}=P_{j}(r) e^{i k \varphi}$ to construct the region-based RI descriptors on the frequency domain (please refer to [27] about specific parameter settings of convolution kernels in detail). Therefore, the resulting final $\mathrm{SFCF}$ is

$$
\begin{aligned}
& F_{\mathrm{SFCF}}=\left[\Omega_{1}(\mathbf{I})_{C_{1}}, \ldots, \Omega_{1}(\mathbf{I})_{C_{j}}, \ldots, \Omega_{2}(\mathbf{I})_{C_{1}}, \ldots\right. \\
&\left.\times \Omega_{2}(\mathbf{I})_{C_{j}}, \ldots, \Omega_{3}(\mathbf{I})_{C_{1}}, \ldots, \Omega_{3}(\mathbf{I})_{C_{j}}, \ldots\right]
\end{aligned}
$$

where $\Omega_{i}(\mathbf{I})_{C_{j}}$ is the region-based features using the $j$ th convolution kernel.

\section{B. Feature Learning or Refine}

To effectively eliminate the feature gap between the two different domains and, meanwhile, to improve its robustness and representative ability, we are able to learn or refine the feature cube (see Fig. 6) along the spatial and channel directions using the following two strategies.

Module 1 [Subspace-Based Learning (e.g., Principal Component Analysis [53])]: The extracted SFCF features can be further learned to reduce the computational and storage cost as well as improve the feature representation ability to some extent.

Module 2 (Aggregation-Based Pooling): The SFCF can also be defined by the pooling-like operation (ACF) to dynamically adjust the support regions with different sizes and, meanwhile, maintain the structural consistency with the overall image [31].

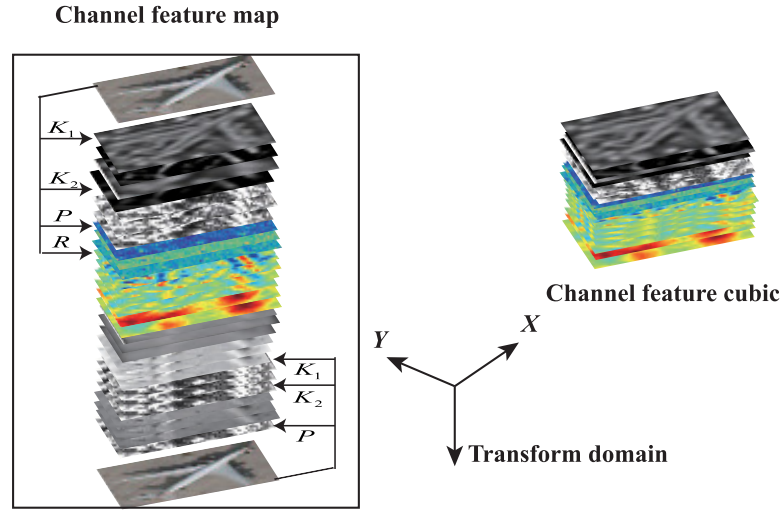

Fig. 6. SFCFs of an object sample and its region-based feature cubic.

Subsequently, the 2-D ACF is stretched to the 1-D fully connected feature vector, making it better fitting into ensemble classifier learning. Inspired by the structurally encoding pattern, we select the ACF during the process of feature refine.

\section{Training Phase With Ensemble Classifier Learning}

Up to the present, boosting is one of the most popular learning techniques by integrating a large number of weak learners to generate a stronger one. The boosting-based method (e.g., AdaBoost) is built on the fact that those selected week classifiers should minimize the training errors and keep or reduce the test errors. For this reason, we apply a softcascade boosting structure with the depth-3 decision trees [28], which is the capability of discriminating intrasamples and intersamples more effectively and simultaneously playing a role in feature selection. Significantly, the learning strategy is robust against background interference in object detection, especially in a more complex scene of remote sensing imagery.

\section{Test Phase With Feature Channel Scaling}

The sliding window is a commonly used detection technique in the testing phase behind extracting finely sampled image pyramid. However, it implies a heavy computational cost, which is not a good tool in the real world. A fast image pyramid model [28] introduced in Section II-B is implemented in our framework by automatically estimating the scaling factor of feature channels, which is expressed as

$$
\mathbf{C}(\mathbf{I}, s) \approx \Omega(\mathbf{R}(\mathbf{I}, s))=\mathbf{R}(\mathbf{I}, s) \cdot s^{-\lambda_{\Omega}}
$$

where $\mathbf{I}$ is the input image and $\mathbf{R}(\mathbf{I}, s)$ is a resampled image of $\mathbf{I}$ by $s . \lambda$ is a scaling factor to be estimated. The corresponding channel image at a scale $s$ can be presented by (12). The different channels can be computed with a linear or nonlinear transformation of the original image in the spatial and frequency domains. Using (12), we can quickly obtain the channels features of all pyramid images using the given $\lambda$ value calculated in the training phase.

\section{EXPERIMENTAL RESUlTS AND ANALYSIS}

\section{A. Optical Remote Sensing Data Sets}

In this section, two well-known public optical remote data sets, car targets in satellite data $\operatorname{set}^{3}$ and airplane targets in

\footnotetext{
${ }^{3}$ http://ai.stanford.edu/ gaheitz/Research/TAS/tas.v0.tgz
} 
NWPU VHR-airplane data set, ${ }^{4}$ are used to quantitatively evaluate the performances of the proposed method. In this paper, $60 \%$ samples are assigned as the training set, and the rest is the testing set for both the data sets. The main focus of this paper is to create a more robust and discriminative feature representation, ensuring rotation and translation invariance. Generally, it is very expensive and time-consuming to collect a large number of training samples, particularly labeling remote sensing data. Therefore, it is very meaningful and challenging for users to assess the generalization performance of the classifier with a limited training set. To stably evaluate the performance of the proposed method, we conduct fivefold cross validation and report an average result below across the folds.

1) Satellite Data Set: This data set was acquired from Google Earth [54]. The low resolution and the varying illumination conditions caused by the shadows of buildings make this data set very challenging. In detail, the images contain 1319 manually labeled cars from 30 images with a size of $792 \times 636$. At the training stage, all car windows are rescaled to $40 \times 40$, due to the average car window that is approximately this window. Also, their mirror images are used to double the positive images as data augmentation in all experiments, which can avoid overfitting and improve the generalization ability. Meanwhile, the negative images are cropped at random positions from the 226 natural images without any car objects.

2) NWPU VHR-10 Data Set: This data set consists of ten different object detection data sets acquired from Google Earth (a spatial resolution of $0.5-2 \mathrm{~m}$ ) and the Vaihingen data set (a spatial resolution of $0.08 \mathrm{~m})^{5}$ Please refer to [24] and [25] for more details. To meet our experimental assumption, that is, we mainly aim at detecting those objects with highly rotation behavior, the airplane is the proper research object, which is selected as our another experimental data to effectively evaluate our method. More specifically, the positive image set without any outliers is composed of 650 airplane images, and each of them includes at least one target. The negative image set consists of 150 images without any class-relevant targets. The original maximal and minimal windows are set to $130 \times 120$ and $40 \times 40$ pixels, respectively. Additionally, the number of positive images in the training set is doubled by mirror processing, while the negative images are randomly selected from the 100 images without any airplanes.

\section{B. Experimental Setup}

All the experiments in this paper were implemented with Matlab2016 on a Windows 7 operation system and conducted on an Intel Xeon 2.6 GHz PC (CPU) with 128-GB memory. Moreover, there are several important modules in the proposed ORSIm framework, such as SFCF extraction, sampling window, smoothing, feature pyramid, and classifier setting. We will gradually detail them in the following.

1) SFCF Extraction: The channel features used in our case mainly consist of two parts: spatial channel features and frequency channel features. The former involves color channels

\footnotetext{
${ }^{4}$ http://www.ifp.uni-stuttgart.de/dgpf/DKEPAllg.html.

${ }^{5}$ The Vaihingen data were provided by the DGPF.
}

and the corresponding magnitude of gradient channels, and the latter is the RI feature channels. More specifically, RGB, LUV, and HSV are selected as the potential color spaces. The magnitude of the gradient channel is set as the magnitude of the channel with the maximal gradient amplitude response. There are three parts in the RI channels, which are the true RI features (the same Fourier orders, e.g., $m_{1}+m_{2}=0$ ), the magnitude features, and the coupling features across different radii (please refer to [27] for more details). During the process, two parameters need to be considered, namely the radii $(r)$ of convolutional kernels and the number of Fourier order $(m)$. We assign five scales with six different half-widths of $\sigma=\{3,4,5,6,7,8\}$ to the value of $r$, i.e., $\sigma=6, r_{j} \in$ $\{0,6,12,18,24\}$, while $m$ is set to $2,3,4$, and 5 , as suggested in [27].

2) Sampling Window: Due to the fact that objects in a scene hold the different resolution, it is necessary for objects (e.g., vehicle and airplane) to be upsampled or downsampled to a consistent size. Therefore, we attempt to search an optimal length-width ratio in a proper range, by resizing the cars on the satellite data set to $28 \times 24,32 \times 28,40 \times 36$, and $44 \times 40$, as well as the airplanes on the NWPU VHR-airplane data set to $56 \times 56,64 \times 64,72 \times 72,80 \times 80$, and $88 \times 88$.

3) Smoothing: The smoothing operation has been proven to be effective in improving the representation ability of the features [28], [31]. Similarly, we perform smoothing before feature computation (pre-smoothing) and after feature learning or refine (post-smoothing) with the binomial filter. The filter radius is set to 1 in our setting.

4) Feature Pyramid: The fast feature pyramid in [28] is applied in the proposed ORSIm framework by coarsely sampling feature channels in order to speed up the hard negative mining and the test phase without additional loss of detection precision. We sample the objects in the four different scales $(s=1,2,4,8)$ with the sampling rate of $2^{-(1 / n P e r O c t)}$. The smallest pyramid image is determined by the size of sampling window, and the largest one has the same size as the original image.

5) Classifier Setting: AdaBoost [55], which is a boostingbased ensemble classifier learning, is used to train the classifier. To train a stronger learner, we use a weighted majority voting to generate the boosting decision tree by combining the hypotheses obtained from those diversified weaker learners. To avoid overfitting, we gradually increase the number of weak learners from 32 to 2048. It is worth noting that the negative samples used in the training phase and the testing phase are selected using a sliding window and a coarsely sampled image pyramid instead of point-based operators as presented in [27].

6) Evaluation Criteria: Four criteria, precision-recall (PR) curve, average precision (AP), average recall (AR), and average F1-score (AF), are adopted to quantitatively evaluate the detection performances. More precisely, when the rate between the overlap of the detection bounding box and the ground-truth box exceeds $50 \%$, it is counted as a true positive (TP); otherwise, as a false negative (FN). Therefore, the final precision $(\mathrm{P})$ is computed by (TP/TP + FP), and the recall (R) is computed by (TP/TP + FN), while F1-score can be computed by 
TABLE I

Performance Comparisons of Eight Different Methods in Terms of AP, AR, And AF. The Best Results Are Shown in Bold

\begin{tabular}{l||c||c|c|c|c|c|c|c|c}
\hline \multirow{2}{*}{ Method } & \multirow{2}{*}{ Image pyramid } & \multicolumn{4}{|c|}{ Satellite (\%) } & \multicolumn{3}{c}{ NWPU VHR-Airplane (\%) } \\
\cline { 3 - 9 } & & AR & AF & AP & FPS & AR & AF & AP & FPS \\
\hline \hline Exemplar-SVMs & Standard pyramid & 78.29 & 81.62 & 85.25 & 0.92 & 80.28 & 80.32 & 80.37 & 0.67 \\
Rotation-aware & Standard pyramid & 77.64 & 78.80 & 80.01 & 1.28 & 81.76 & 80.54 & 79.35 & 0.61 \\
COPD-based & Standard pyramid & 76.21 & 78.23 & 80.37 & 1.05 & 75.17 & 79.84 & 85.13 & 1.06 \\
BOW-SVM & Standard pyramid & 8.29 & 10.77 & 15.38 & 1.16 & 3.58 & 6.27 & 25.12 & 1.17 \\
YOLO2 $(\mathrm{GPU})$ & - & 83.25 & 85.70 & 88.30 & 9.12 & 84.92 & 87.27 & 89.75 & 9.13 \\
FourierHOG & Standard pyramid & 88.20 & 89.78 & 91.42 & 0.83 & 87.78 & 88.97 & 90.20 & 0.66 \\
ACF & fast pyramid & 62.14 & 68.59 & 76.53 & 8.98 & 62.56 & 64.04 & 65.59 & 8.05 \\
ORSIm Detector & fast pyramid & $\mathbf{9 1 . 2 6}$ & $\mathbf{9 3 . 0 1}$ & $\mathbf{9 4 . 8 3}$ & 4.94 & $\mathbf{9 1 . 1 2}$ & $\mathbf{9 3 . 2 1}$ & $\mathbf{9 5 . 3 9}$ & 4.72 \\
\hline
\end{tabular}

$F 1=(2 \times P \times R / P+R)$. AP is used as a global indicator to assess the performances of the algorithm.

\section{Experimental Results}

1) Discussion on Classifier Selection: As listed in Table II, those methods based on SVMs or random forest (RF) classifier also achieve the good performances. This motivates us to have a great interest in investigating the classifier selection. To this end, three different classifiers (e.g., linear SVM, RF, and AdaBoost $)^{6}$ are used to evaluate the detection performance under four different feature descriptors, that is, HOG, ACF, FourierHOG, and our SFCF, as detailed in Table II. For a fair comparison, the parameters used in the three classifiers are optimally tuned by cross validation on the training set. Overall, the linear SVM yields the relatively poor performances compared with the results of RF because the RF is more robust than the linear SVM to some extent, especially when the training samples are limited. Furthermore, the AdaBoost performs better than the two other classifiers. Two possible factors could explain the results. On one hand, AdaBoost is a boosting-based ensemble classifier learning, which can generate a more robust strong classifier by weighing a large number of weak classifiers. Consequently, it holds a more powerful performance than the linear SVM in recognition and classification. On the other hand, although both the RF and AdaBoost are based on the boostinglike strategy, yet the RF equally puts the weights on each subclassifier and the AdaBoost adaptively weighs each weak classifier by iteratively updating weights. This makes the resulting final classifier generated by Adaboost more suitable for the current data set, thereby yielding a better performance.

2) Overview of Performance Comparison: To quantitatively assess the detection performances of the proposed method, we compare several state-of-the-art methods related to our framework, such as exemplar-SVMs [56], rotation-aware features [57], collection of part detectors-based [24], bag-ofwords with SVM (BOW-SVM) [58], fast feature pyramids [28], you only look once (YOLO2) [59], ${ }^{7}$ and FourierHOG [27]. ${ }^{8}$ Fig. 7 shows the PR curves of different algorithms on the two data sets, and Table I correspondingly

\footnotetext{
${ }^{6}$ AdaBoost [55], also known as AdaBoost-DTree, is used in our framework.

${ }^{7}$ Similar to [25] and [60], data augmentation by the rotation and translation of the training samples is performed.

${ }^{8}$ We select positive and negative samples by sliding windows rather than points for a fair comparison.
}

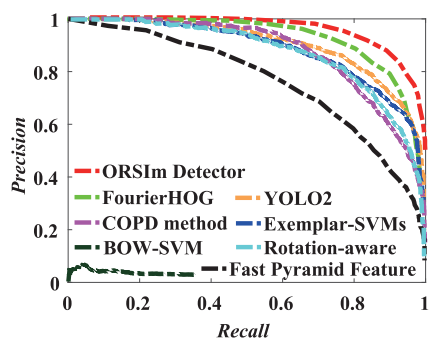

(a)

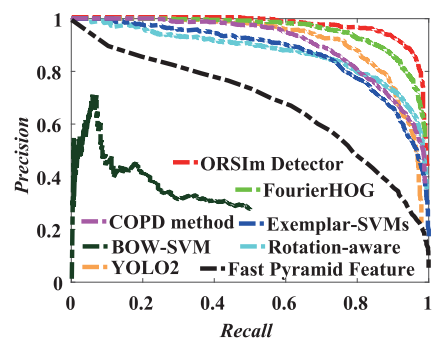

(b)
Fig. 7. PR curves of the proposed ORSIm detector in comparison with the state-of-the-art approaches. (a) Satellite data set. (b) NWPU VHRAirplane.

lists the quantitative results in terms of APs and mean running times. Accordingly, we can make the following observations. The exemplar-SVMs and rotation-aware methods have similar performances, as the standard HOG features and discrete grid sampling are used. Not surprisingly, BOW-SVM and ACF yield the worst performances because they ignore the spatial contextual relationships among the local features and are limited by the rotation-related representation ability. Although the detection performance might be improved by modeling a deeper network and embedding anchor boxes, yet YOLO2 is not robust to tiny object and arbitrary pairs of objects that are not more than a tiny distance apart. FourierHOG holds a slightly lower performance than ours but much better than others on the two data sets, which indicates that the point-based feature representation is insensitive to resolution. As expected, the proposed ORSIm detector largely outperforms the other investigated methods on both the data sets, which shows its effectiveness and superiority. This can also be demonstrated in Table II that the precision of the ORSIm detector is dramatically higher than that of the others owing to the well-designed SFCF and the use of AdaBoost. It is worth noting in Table I that the methods with fast feature pyramid allow for faster detection than those without it. Despite of slowing down the speed (relatively lower than ACF and YOLO2), ${ }^{9}$ the proposed ORSIm detector acquires the highest detection precision.

Visually, a few roofs are wrongly identified as cars, and there is also some leak detection in transport cars, as shown in the first row of Fig. 8. This might result from a limited

\footnotetext{
${ }^{9}$ The code is run on the tensorflow using GPU, which is available from the website: https://github.com/simo23/tinyYOLOv2.
} 

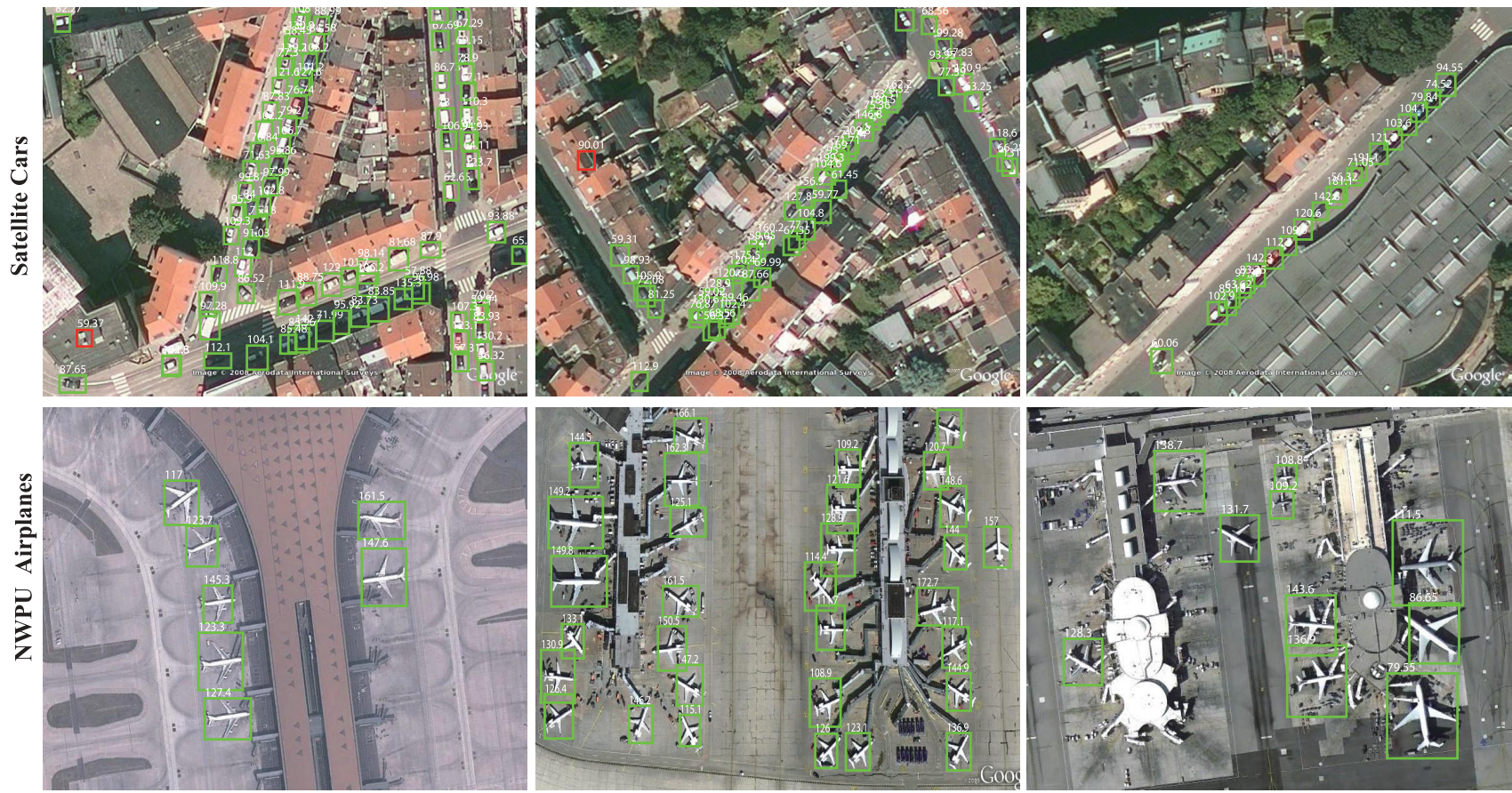

Fig. 8. Some visual detection results (false detection in red box, TP in green box, and missing detection in blue box) by using the proposed method on the two different data sets.

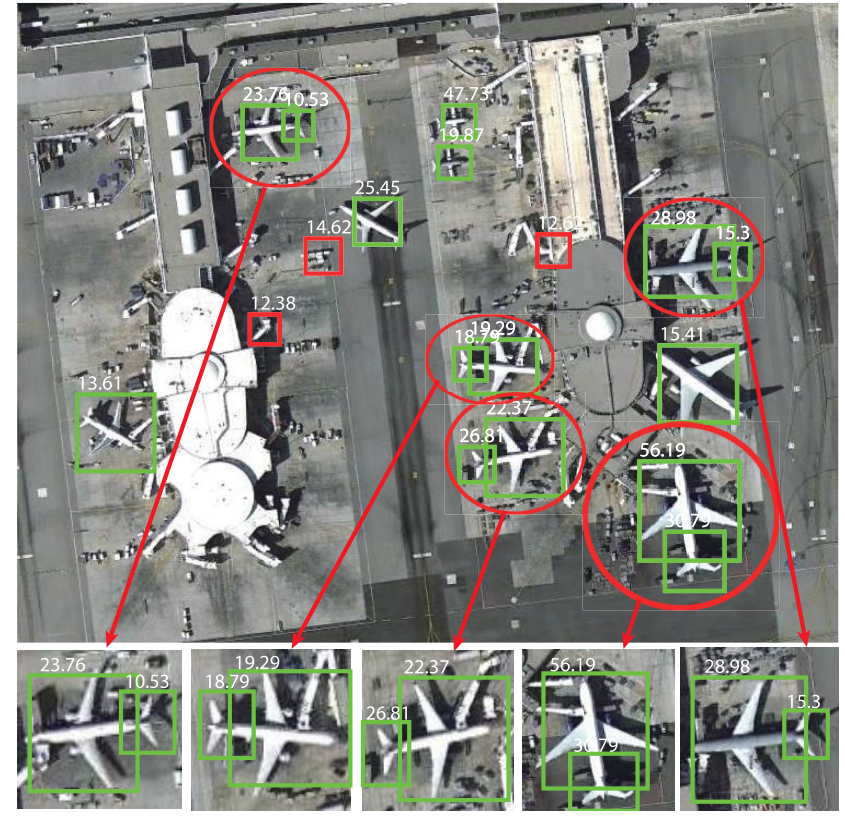

(a)

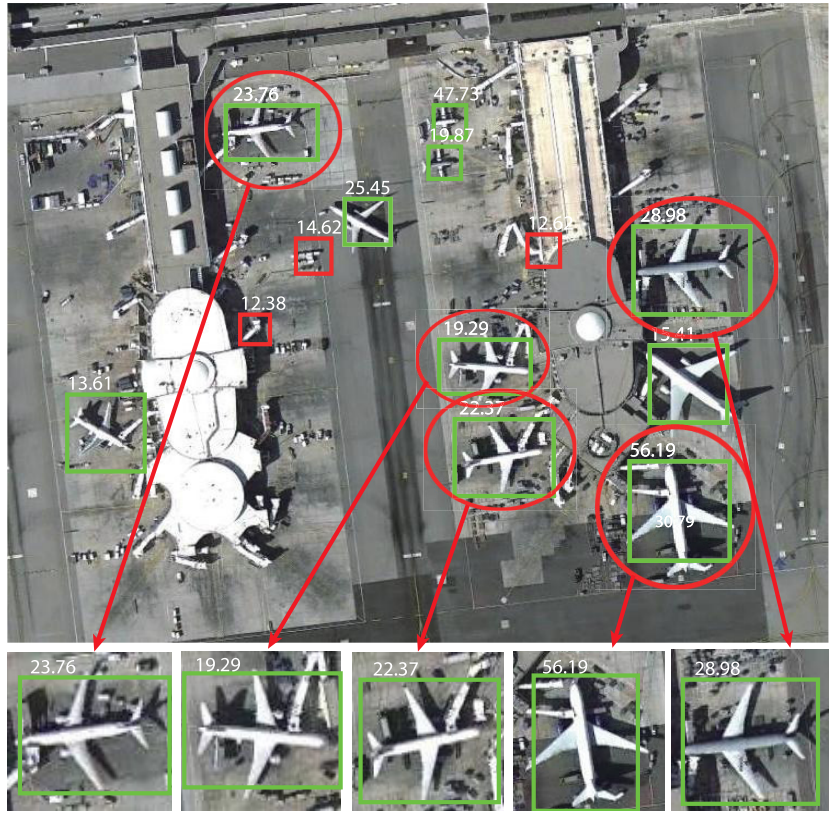

(b)

Fig. 9. Overlap removal using original NMS and two-step NMS. (a) Original NMS. (b) Two-step NMS.

number of training samples and unbalanced class distribution. In addition, a weaker visible edge might mislead the classifier since the transport cars are white. Compared with car detection in a complex urban scene, false detection of the airplanes also occurs when the background and targets have similar shape and color, i.e., the tail of the airplane [see Fig. 9(a)]. But this issue can be well fixed by a two-step nonmaximum suppression (NMS) algorithm [36]. The improved results can be found in Fig. 9(b).

\section{Sensitivity Analysis}

We experimentally analyze and discuss the potential influences under the different configuration of the proposed ORSIm detector, making it possible to generalize well in more data sets. The optimal combination is finally determined by fivefold cross validation on the training set.

1) Toward Parameter Setting: Figs. 10 and 11 show the performance comparison of the different parameter setting on the two used data sets. More specifically, the LUV color 

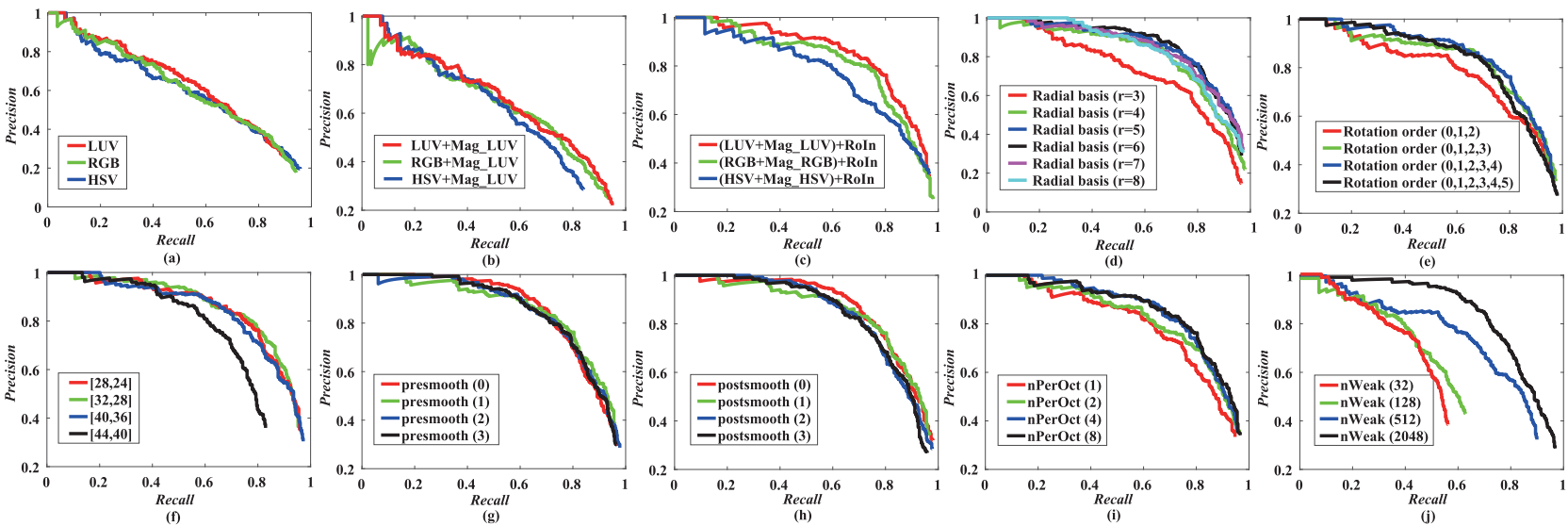

Fig. 10. Performance comparison of the ORSIm detector under different parameter settings on the satellite data set.
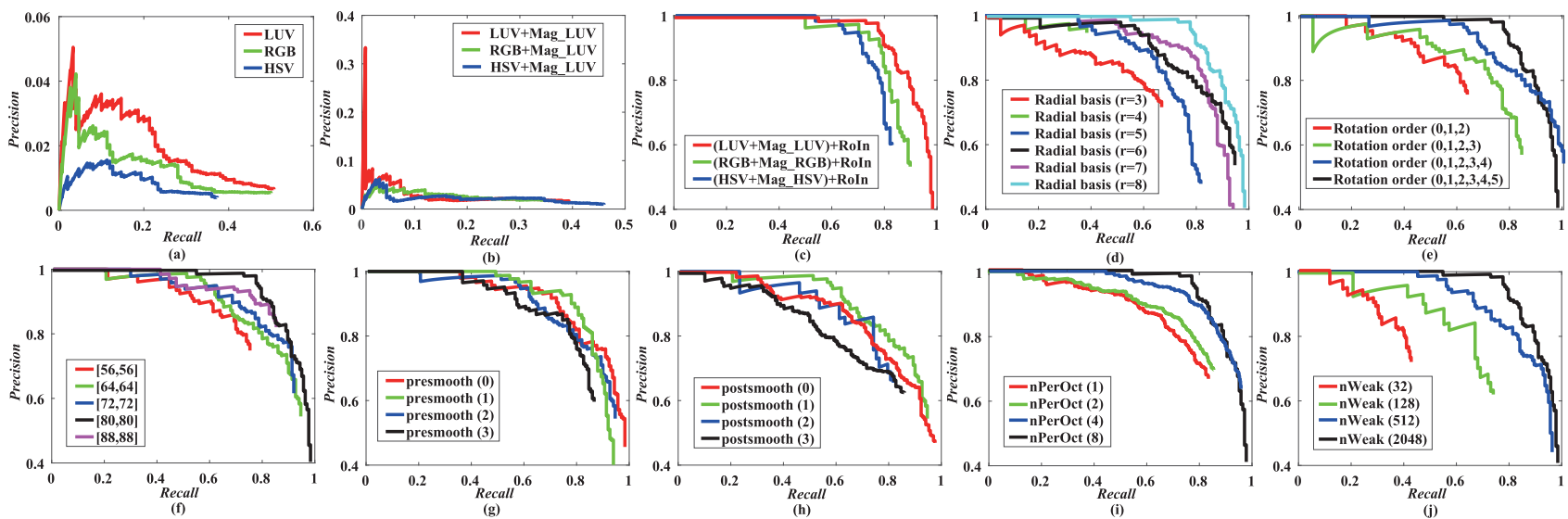

Fig. 11. Performance comparison of the ORSIm detector under different parameter settings on the NWPU VHR-Airplane data set.

TABLE II

Performance Comparisons of Three Different Classifiers. The Best Results Are Shown in Bold

\begin{tabular}{l||c|c|c|c|c}
\hline Dataset & Method & HOG [35] & ACF [28], [30] & FourierHOG & our SFCF \\
\hline \hline \multirow{3}{*}{ Satellite } & Linear SVM & 65.30 & 68.72 & 83.19 & 85.18 \\
& RF & 71.20 & 73.12 & 86.77 & 88.29 \\
& AdaBoost & 74.38 & 76.53 & 90.42 & $\mathbf{9 3 . 1 2}$ \\
\hline \multirow{3}{*}{ NWPU VHR-Airplane } & Linear SVM & 74.38 & 76.67 & 80.01 & 85.12 \\
& RF & 70.05 & 74.98 & 87.26 & 91.68 \\
& AdaBoost & 77.98 & 80.37 & 90.20 & $\mathbf{9 4 . 3 1}$ \\
\hline
\end{tabular}

space performs better than the two others on both the data sets and even more obvious when using a combination of the color channels with GM channel. Interestingly, there is a similar trend after adding the RI feature channels, as shown in Figs. 10 and 11(c).

We also investigate the effects of the radial profiles (the size of convolution kernel) and the Fourier orders $(k \in\{0 \sim m\})$ as well as the size of sampling windows. As observed in Figs. 10 and 11(d) and (e), they are relatively insensitive in a proper range, and as a result, we select them as $m=4$ for both the data sets and $r=6,32 \times 28$ for the satellite data set $(r=8$, $80 \times 80$ for the NWPU VHR-airplane data set). Following the same strategy with the traditional detection framework, pre-smoothing and post-smoothing are usually carried out before and after running the detection algorithms in order to make the feature locally and globally smooth. The different filter radii $\in\{0,1,2,3\}$ are selected for smoothing, and the experimental results are given in Figs. 10 and $11(\mathrm{~g})$ and $(\mathrm{h})$. We simply set the radius for both pre-smoothing and postsmoothing as 1 , as they are relatively insensitive to the different radius. In the test phase, the pyramid factor plays an important role, as shown in Figs. 10 and 11(i). The eight scales per octave show the best result, which is basically consistent with [28]. Significantly, the final detection precision would increase with the number of the weak classifier but so does the computational cost. As a tradeoff, the value is set as 2048 in our case.

2) Toward Spatial Resolution: The image resolution is another important factor that could degrade the detection performance, and therefore, we emphatically evaluate the effects 


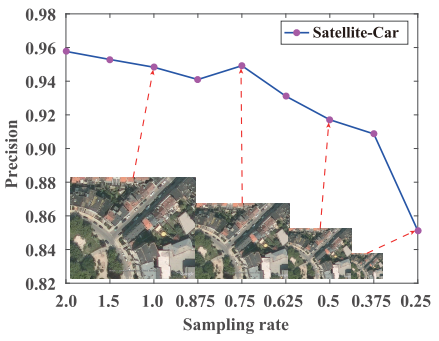

(a)

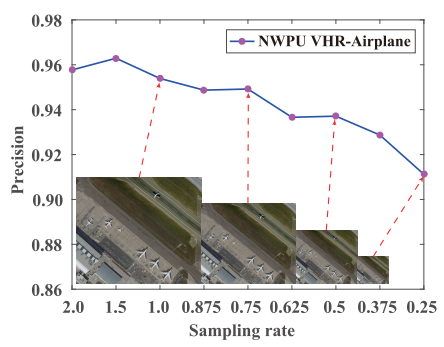

(b)
Fig. 12. Sensitivity investigation to different spatial resolutions of the image. (a) Vehicle data set. (b) Airplane data set.

of different resolutions to find a proper boundary condition for the use of the proposed ORSIm detector. In detail, we adopt the different sampling rates on the two data sets to investigate the sensitivity of detection precision. As can be seen from Fig. 12, the performance may begin to degenerate with around 0.5 sampling rate and gradually decrease after that. It should be noted that the feature pyramid is usually an indispensable step in the test phase. Therefore, these detection approaches are, in fact, not so sensitive to different spatial resolutions, although the lower resolution inevitably suffers from information loss. Furthermore, Fig. 8 shows a visual example to clarify that the different scaled objects can be basically detected, demonstrating the effectiveness of the ORSIm detector to the multiresolution images. That is not to say, however, that the proposed detector is capable of handling various variations. For that, we highlight a scene to give some false cases, as shown in Fig. 9, where the detector confuses the real airplanes and its tails with a small shadow, leading to some extra false alarms marked in red color. This is actually a comparatively common phenomenon in object detection rather than due to the model's sensitivity to the spatial resolution of an input image [36]. A feasible solution for this issue is to use a two-step NMS, as shown in Fig. 9.

\section{CONCLUSION}

Object rotation is a common but challenging issue for object detection and recognition in optical remote sensing. To this end, we propose a more complete object detection framework in ORSIm, called the ORSIm detector, by introducing the discriminative RI channel features (SFCFs), learning-based feature refining, and fast feature channel scaling technique as well as boosting-based classifier learning. The extensive experimental results indicate that the ORSIm detector performs better and is more robust to various deformations compared with the previous state-of-the-art methods. In future work, we will focus on tiny object detection and extend the proposed framework to an end-to-end learning framework (e.g., deep learning). Additionally, we will expand the binary classification to multitarget detection.

\section{ACKNOWLEDGMENT}

The authors would like to thank the Key Laboratory of Information Fusion Technology, Ministry of Education, University of Northwestern Polytechnical, and the Electrical
Engineering Department, Stanford University, for providing the NWPU VHR-airplane data set and the Satellite data set. They would also like to thank Prof. P. Dollár and Dr. K. Liu for providing MATLAB codes for fast pyramid feature and FourierHOG algorithms.

\section{REFERENCES}

[1] D. Hong, N. Yokoya, and X. X. Zhu, "Learning a robust local manifold representation for hyperspectral dimensionality reduction," IEEE J. Sel. Topics Appl. Earth Observ. Remote Sens., vol. 10, no. 6, pp. 2960-2975, Jun. 2017

[2] D. Hong, N. Yokoya, J. Xu, and X. Zhu, "Joint \& progressive learning from high-dimensional data for multi-label classification," in Proc. IEEE Conf. Eur. Comput. Vis. (ECCV), Sep. 2018, pp. 478-493.

[3] Z. Hu, Q. Li, Q. Zou, Q. Zhang, and G. Wu, "A bilevel scale-sets model for hierarchical representation of large remote sensing images," IEEE Trans. Geosci. Remote Sens., vol. 54, no. 12, pp. 7366-7377, Dec. 2016.

[4] Z. Hu, Q. Li, Q. Zhang, Q. Zou, and Z. Wu, "Unsupervised simplification of image hierarchies via evolution analysis in scale-sets framework," IEEE Trans. Image Process., vol. 26, no. 5, pp. 2394-2407, May 2017.

[5] S. Henrot, J. Chanussot, and C. Jutten, "Dynamical spectral unmixing of multitemporal hyperspectral images," IEEE Trans. Image Process., vol. 25, no. 7, pp. 3219-3232, Jul. 2016.

[6] D. Hong, N. Yokoya, J. Chanussot, and X. X. Zhu, "Learning a lowcoherence dictionary to address spectral variability for hyperspectral unmixing," in Proc. IEEE Int. Conf. Image Process. (ICIP), Sep. 2017, pp. $235-239$.

[7] D. Hong and X. X. Zhu, "SULoRA: Subspace unmixing with low-rank attribute embedding for hyperspectral data analysis," IEEE J. Sel. Topics Signal Process., vol. 12, no. 6, pp. 1351-1363, Oct. 2018.

[8] D. Hong, N. Yokoya, J. Chanussot, and X. X. Zhu, "An augmented linear mixing model to address spectral variability for hyperspectral unmixing," IEEE Trans. Image Process., vol. 28, no. 4, pp. 1923-1938, Apr. 2019.

[9] M. A. Veganzones, M. Simões, G. Licciardi, N. Yokoya, J. M. Bioucas-Dias, and J. Chanussot, "Hyperspectral super-resolution of locally low rank images from complementary multisource data,' IEEE Trans. Image Process., vol. 25, no. 1, pp. 274-288, Jan. 2016.

[10] D. Hong, N. Yokoya, J. Chanussot, and X. X. Zhu. (2018). "Cospace: Common subspace learning from hyperspectral-multispectral correspondences." [Online]. Available: https://arxiv.org/abs/1812.11501

[11] D. Hong, N. Yokoya, N. Ge, J. Chanussot, and X. X. Zhu, "Learnable manifold alignment (LeMA): A semi-supervised cross-modality learning framework for land cover and land use classification," ISPRS J. Photogramm. Remote Sens., vol. 147, pp. 193-205, Jan. 2019.

[12] N. Yokoya and A. Iwasaki, "Object detection based on sparse representation and Hough voting for optical remote sensing imagery," IEEE J. Sel. Topics Appl. Earth Observ. Remote Sens., vol. 8, no. 5, pp. 2053-2062, May 2015.

[13] G. Cheng and J. Han, "A survey on object detection in optical remote sensing images," ISPRS J. Photogramm. Remote Sens., vol. 117, pp. 11-28, Jul. 2016.

[14] G. Tochon, J. Chanussot, M. D. Mura, and A. L. Bertozzi, "Object tracking by hierarchical decomposition of hyperspectral video sequences: Application to chemical gas plume tracking," IEEE Trans. Geosci. Remote Sens., vol. 55, no. 8, pp. 4567-4585, Aug. 2017.

[15] D. Hong, Z. Pan, and X. Wu, "Improved differential box counting with multi-scale and multi-direction: A new palmprint recognition method," OPTIK, vol. 125, no. 15, pp. 4154-4160, Aug. 2014.

[16] M. Zhang, W. Li, and Q. Du, "Diverse region-based CNN for hyperspectral image classification," IEEE Trans. Image Process., vol. 27, no. 6, pp. 2623-2634, Jun. 2018.

[17] X. Xu, W. Li, Q. Ran, Q. Du, L. Gao, and B. Zhang, "Multisource remote sensing data classification based on convolutional neural network," IEEE Trans. Geosci. Remote Sens., vol. 56, no. 2, pp. 937-949, Feb. 2018.

[18] D. Hong, W. Liu, X. Wu, Z. Pan, and J. Su, "Robust palmprint recognition based on the fast variation Vese-Osher model," Neurocomputing, vol. 174, pp. 999-1012, Jan. 2016.

[19] F. Maussang, J. Chanussot, A. Hétet, and M. Amate, "Higher-order statistics for the detection of small objects in a noisy background application on sonar imaging," EURASIP J. Adv. Signal Process., vol. 2007, no. 1, p. 25, 2007.

[20] H. Grabner, T. T. Nguyen, B. Gruber, and H. Bischof, "On-line boostingbased car detection from aerial images," ISPRS J. Photogramm. Remote Sens., vol. 63, no. 3, pp. 382-396, May 2008. 
[21] T. Moranduzzo and F. Melgani, "Automatic car counting method for unmanned aerial vehicle images," IEEE Trans. Geosci. Remote Sens., vol. 52, no. 3, pp. 1635-1647, Mar. 2014.

[22] C. Corbane, L. Najman, E. Pecoul, L. Demagistri, and M. Petit, "A complete processing chain for ship detection using optical satellite imagery," Int. J. Remote Sens., vol. 31, no. 22, pp. 5837-5854, Dec. 2010.

[23] C. Zhu, H. Zhou, R. Wang, and J. Guo, "A novel hierarchical method of ship detection from spaceborne optical image based on shape and texture features," IEEE Trans. Geosci. Remote Sens., vol. 48, no. 9, pp. 3446-3456, Sep. 2010.

[24] G. Cheng, J. Han, P. Zhou, and L. Guo, "Multi-class geospatial object detection and geographic image classification based on collection of part detectors," ISPRS J. Photogramm. Remote Sens., vol. 98, no. 1, pp. 119-132, Dec. 2014.

[25] G. Cheng, P. Zhou, and J. Han, "Learning rotation-invariant convolutional neural networks for object detection in VHR optical remote sensing images," IEEE Trans. Geosci. Remote Sens., vol. 54, no. 12, pp. 7405-7415, Dec. 2016.

[26] P. Viola and M. J. Jones, "Robust real-time face detection," Int. J. Comput. Vis., vol. 57, no. 2, pp. 137-154, May 2004.

[27] K. Liu et al., "Rotation-invariant HOG descriptors using Fourier analysis in polar and spherical coordinates," Int. J. Comput. Vis., vol. 106, no. 3, pp. 342-364, Feb. 2014.

[28] P. Dollár, R. Appel, S. Belongie, and P. Perona, "Fast feature pyramids for object detection," IEEE Trans. Pattern Anal. Mach. Intell., vol. 36, no. 8, pp. 1532-1545, Aug. 2014.

[29] Y. Freund and R. E. Schapire, "A decision-theoretic generalization of on-line learning and an application to boosting," J. Comput. Syst. Sci., vol. 55, no. 1, pp. 119-139, Aug. 1997.

[30] P. Dollar, Z. Tu, and P. Perona, "Integral channel features," in Proc. Brit. Mach. Vis. Conf. (BMVC), Sep. 2009, pp. 1-12.

[31] B. Yang, J. Yan, Z. Lei, and S. Z. Li, "Aggregate channel features for multi-view face detection," in Proc. IEEE Int. Joint Conf. Biometrics (IJCB), Sep./Oct. 2014, pp. 1-8.

[32] S. Wang, B. Yang, Z. Lei, J. Wan, and S. Z. Li, "A convolutional neural network combined with aggregate channel feature for face detection," in Proc. 6th Int. Conf. Wireless, Mobile Multi-Media (ICWMMN), Nov. 2015, pp. 304-308.

[33] R. Brehar, C. Vancea, and S. Nedevschi, "Pedestrian detection in infrared images using aggregated channel features," in Proc. IEEE 10th Int. Conf. Intell. Comput. Commun. Process. (ICCP), Sep. 2014, pp. 127-132.

[34] S. Tuermer, F. Kurz, P. Reinartz, and U. Stilla, "Airborne vehicle detection in dense urban areas using HoG features and disparity maps," IEEE J. Sel. Topics Appl. Earth Observ. Remote Sens., vol. 6, no. 6, pp. 2327-2337, Dec. 2013.

[35] N. Dalal and B. Triggs, "Histograms of oriented gradients for human detection," in Proc. IEEE Comput. Soc. Conf. Comput. Vis. Pattern Recognit. (CVPR), Jun. 2005, pp. 886-893.

[36] A. Zhao et al., "An effective method based on ACF for aircraft detection in remote sensing images," IEEE Geosci. Remote Sens. Lett., vol. 14, no. 5, pp. 744-748, May 2017.

[37] W. Zhang, X. Sun, K. Fu, C. Wang, and H. Wang, "Object detection in high-resolution remote sensing images using rotation invariant parts based model," IEEE Geosci. Remote Sens. Lett., vol. 11, no. 1, pp. 74-78, Jan. 2014.

[38] G. Wang, X. Wang, B. Fan, and C. Pan, "Feature extraction by rotationinvariant matrix representation for object detection in aerial image," IEEE Geosci. Remote Sens. Lett., vol. 14, no. 6, pp. 851-855, Jun. 2017.

[39] D. Hong, W. Liu, J. Su, Z. Pan, and G. Wang, "A novel hierarchical approach for multispectral palmprint recognition," Neurocomputing, vol. 151, pp. 511-521, Mar. 2015.

[40] P. Viola and M. J. Jones, "Rapid object detection using a boosted cascade of simple features," in Proc. IEEE Comput. Soc. Conf. Comput. Vis. Pattern Recognit. (CVPR), Dec. 2001, p. I.

[41] P. Viola, M. J. Jones, and D. Snow, "Detecting pedestrians using patterns of motion and appearance," Int. J. Comput. Vis., vol. 63, no. 2, pp. 153-161, Jul. 2005.

[42] D. L. Ruderman and W. Bialek, "Statistics of natural images: Scaling in the woods," Phys. Rev. Lett., vol. 73, no. 6, pp. 814-817, Aug. 1994.

[43] R. Benenson, M. Mathias, R. Timofte, and L. Van Gool, "Pedestrian detection at 100 frames per second," in Proc. CVPR, Jun. 2012, pp. 2903-2910.

[44] S. Ram and J. J. Rodriguez, "Vehicle detection in aerial images using multiscale structure enhancement and symmetry," in Proc. IEEE Int. Conf. Image Process. (ICIP), Sep. 2016, pp. 3817-3821.
[45] J. Zhang, C. Tao, Z. Zou, and H. Pan, "A vehicle detection method taking shadow areas into account for high resolution aerial imagery," in Proc. IEEE Int. Geosci. Remote Sens. Symp. (IGARSS), Jul. 2016, pp. 669-672.

[46] M. Alb, P. Alotto, G. Capasso, M. Guarnieri, C. Magele, and W. Renhart, "Real-time pose detection for magnetic-assisted medical applications by means of a hybrid deterministic/stochastic optimization method," IEEE Trans. Magn., vol. 52, no. 3, Mar. 2016, Art. no. 5100104.

[47] X. Nie, J. Feng, J. Xing, S. Xiao, and S. Yan, "Hierarchical contextual refinement networks for human pose estimation," IEEE Trans. Image Process., vol. 28, no. 2, pp. 924-936, Feb. 2019.

[48] J. Xia, J. Chanussot, P. Du, and X. He, "Rotation-based support vector machine ensemble in classification of hyperspectral data with limited training samples," IEEE Trans. Geosci. Remote Sens., vol. 54, no. 3, pp. 1519-1531, Mar. 2016

[49] A. Vedaldi, M. Blaschko, and A. Zisserman, "Learning equivariant structured output SVM regressors," in Proc. IEEE Int. Conf. Comput. Vis. (ICCV), Nov. 2011, pp. 959-966.

[50] J. Xia, P. Du, X. He, and J. Chanussot, "Hyperspectral remote sensing image classification based on rotation forest," IEEE Geosci. Remote Sens. Lett., vol. 11, no. 1, pp. 239-243, Jan. 2014.

[51] P. Dollér, S. Belongie, and P. Perona, "The fastest pedestrian detector in the west," in Proc. Int. Conf. Brit. Mach. Vis. Conf. (BMVC), Dec. 2010 pp. $1-11$.

[52] G. B. Giannakis, "Signal reconstruction from multiple correlations: Frequency- and time-domain approaches," J. Opt. Soc. Amer. A, Opt. Image Sci., vol. 6, no. 5, pp. 682-697, Feb. 1989.

[53] S. T. Roweis and L. K. Saul, "Nonlinear dimensionality reduction by locally linear embedding," Science, vol. 290, no. 5500, pp. 2323-2326, Dec. 2000.

[54] G. Heitz and D. Koller, "Learning spatial context: Using stuff to find things," in Proc. Eur. Conf. Comput. Vis. (ECCV), vol. 5302, Oct. 2008, pp. $30-43$.

[55] J. Friedman, T. Hastie, and R. Tibshirani, "Additive logistic regression: A statistical view of boosting," Ann. Statist., vol. 28, no. 2, p. 337-374, Apr. 2000.

[56] T. Malisiewicz, A. Gupta, and A. A. Efros, "Ensemble of exemplarSVMs for object detection and beyond," in Proc. Int. Conf. Comput. Vis. (ICCV), Nov. 2011, pp. 89-96.

[57] U. Schmidt and S. Roth, "Learning rotation-aware features: From invariant priors to equivariant descriptors," in Proc. IEEE Conf. Comput. Vis. Pattern Recognit. (CVPR), vol. 157, Jun. 2012, pp. 2050-2057.

[58] S. Xu, T. Fang, D. Li, and S. Wang, "Object classification of aerial images with bag-of-visual words," IEEE Geosci. Remote Sens. Lett., vol. 7, no. 2, pp. 366-370, Apr. 2010

[59] J. Redmon and A. Farhadi, "Yolo9000: Better, faster, stronger," in Proc. IEEE Int. Conf. Comput. Vis. Pattern Recognit. (CVPR), Jul. 2017, pp. 7263-7271

[60] X. Wu, D. Hong, P. Ghamisi, W. Li, and R. Tao, "MsRi-CCF: Multi-scale and rotation-insensitive convolutional channel features for geospatial object detection," Remote Sens., vol. 10, no. 12, p. 1990, Dec. 2018.

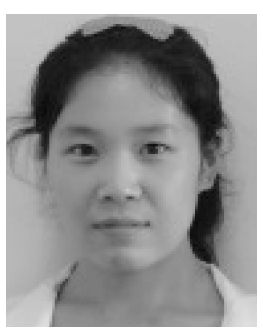

Xin Wu (S'19) received the B.S. degree in science and technology from the Electronic Information Department, Mudanjiang Normal University, Mudanjiang, China, in 2011, and the M.Sc. degree in computer science and technology, Qingdao University, Qingdao, China, in 2014. She is currently pursuing the $\mathrm{Ph} . \mathrm{D}$. degree in information and communication engineering with the Beijing Institute of Technology, Beijing, China.

In 2018, she was a Visiting Student with the Photogrammetry and Image Analysis Department, Remote Sensing Technology Institute, German Aerospace Center, Weßling, Germany, under the supervision of Dr. J. Tian and Prof. P. Reinartz.

Her research interests include signal/image processing, fractional Fourier transform, deep learning, and their applications in biometrics and geospatial object detection. 


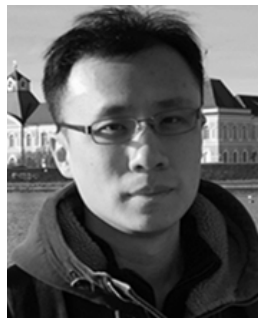

Danfeng Hong (S'16) received the B.Sc. degree in computer science and technology from the Neusoft College of Information, Northeastern University, Shenyang, China, in 2012, and the M.Sc. degree in computer vision from Qingdao University, Qingdao, China, in 2015. He is currently pursuing the Ph.D. degree with Signal Processing in Earth Observation, Technical University of Munich, Munich, Germany.

Since 2015, he has been a Research Associate with the Remote Sensing Technology Institute, German Aerospace Center, Weßling, Germany. In 2018, he was a Visiting Student with GIPSA-Lab, Grenoble INP, CNRS, Université Grenoble Alpes, Grenoble, France, under the supervision of Prof. J. Chanussot.

His research interests include signal/image processing and analysis, pattern recognition, machine/deep learning, and their applications in earth vision.

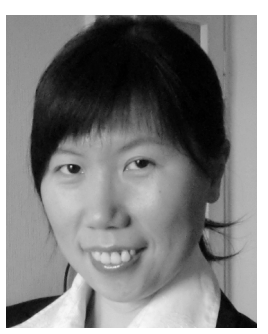

Jiaojiao Tian received the B.S. degree in geoinformation systems from the China University of Geosciences, Beijing, China, in 2006, the M.Eng. degree in cartography and geoinformation from the Chinese Academy of Surveying and Mapping, Beijing, in 2009, and the Ph.D. degree in mathematics and computer science from Osnabrück University, Osnabrück, Germany, in 2013.

Since 2009, she has been with the Photogrammetry and Image Analysis Department, Remote Sensing Technology Institute, German Aerospace Center, Weßling, Germany, where she is currently the Head of the 3D Modeling Team. In 2011, she was a Guest Scientist with the Institute of Photogrammetry and Remote Sensing, ETH Zürich, Zurich, Switzerland. Her research interests include 3-D change detection, digital surface model (DSM) generation and quality assessment, object extraction, and DSM-assisted building reconstruction and classification.

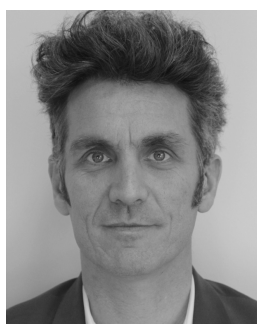

Jocelyn Chanussot (M'04-SM'04-F'12) received the M.Sc. degree in electrical engineering from the Grenoble Institute of Technology (Grenoble INP), Grenoble, France, in 1995, and the Ph.D. degree from the Universit de Savoie, Annecy, France, in 1998.

In 1999, he was with the Geography Imagery Perception Laboratory, Delegation Generale de l'Armement. Since 1999, he has been with Grenoble INP, where he is currently a Professor of signal and image processing and conducting his research with the GIPSA-Lab. He has been a Visiting Scholar with Stanford University, Stanford, CA, USA, the KTH Royal Institute of Technology, Stockholm, Sweden, and the National University of Singapore, Singapore. Since 2013, he has also been an Adjunct Professor with the University of Iceland, Reykjavik, Iceland. From 2015 to 2017, he was a Visiting Professor with the University of California at Los Angeles, Los Angeles, CA, USA. His research interests include image analysis, multicomponent image processing, nonlinear filtering, and data fusion in remote sensing.

Dr. Chanussot was a member of the IEEE Geoscience and Remote Sensing Society AdCom from 2009 to 2010, in charge of membership development. He was a member of the Institut Universitaire de France from 2012 to 2017. He was the Founding President of the IEEE Geoscience and Remote Sensing French Chapter from 2007 to 2010, which received the 2010 IEEE GRSS Chapter Excellence Award. He was a co-recipient of the NORSIG 2006 Best Student Paper Award, the IEEE GRSS 2011 and 2015 Symposium Best Paper Award, the IEEE GRSS 2012 Transactions Prize Paper Award, and the IEEE GRSS 2013 Highest Impact Paper Award. He was the General Chair of the first IEEE GRSS Workshop on Hyperspectral Image and Signal Processing, Evolution in Remote Sensing. He was the Chair from 2009 to 2011 and the Co-Chair from 2005 to 2008 of the GRS Data Fusion Technical Committee. He was a member of the Machine Learning for Signal Processing Technical Committee of the IEEE Signal Processing Society from 2006 to 2008 and the Program Chair of the IEEE International Workshop on Machine Learning for Signal Processing in 2009. He was an Associate Editor of the
IEeE Geoscience and Remote Sensing from 2005 to 2007 and Pattern Recognition from 2006 to 2008. Since 2007, he has been an Associate Editor of the IEEE TRansactions on Geoscience and Remote Sensing. He was a Guest Editor of the PROCEEDINGS OF THE IEEE in 2013 and the IEEE Signal Processing Magazine in 2014. He was the Editor-in-Chief of the IEEE JOURNAL OF SELECTED TOPICS IN APPLIEd EARTH OBSERVATIONS AND REmote SENSING from 2011 to 2015. Since 2018, he has also been an Associate Editor of the IEEE TRANSACTIONS ON IMAGE PROCESSING.

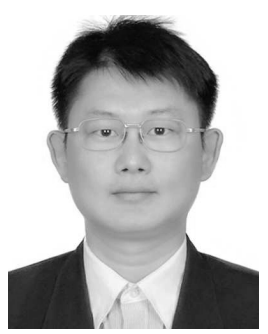

Wei Li (S'11-M'13-SM'16) received the B.E. degree in telecommunications engineering from Xidian University, Xi'an, China, in 2007, the M.S. degree in information science and technology from Sun Yat-sen University, Guangzhou, China, in 2009, and the Ph.D. degree in electrical and computer engineering from Mississippi State University, Starkville, MS, USA, in 2012

He spent one year as a Post-Doctoral Researcher with the University of California at Davis, Davis, CA, USA. He is currently a Professor and the Vice Dean of the College of Information Science and Technology, Beijing University of Chemical Technology, Beijing, China. His research interests include hyperspectral image analysis, pattern recognition, and data compression.

$\mathrm{Dr}$. $\mathrm{Li}$ is an active Reviewer of the IEEE TRANSACTIONS ON GEOSCIENCE and Remote Sensing, the IEEE Geoscience Remote Sensing LetTERS, and the IEEE JOURNAL OF SELECTED TOPICS IN APPLIED EARTH OBSERVATIONS AND REMOTE.

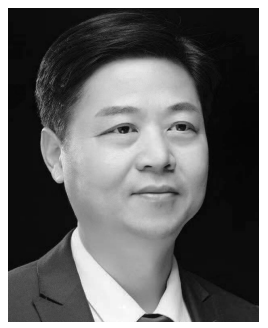

Ran Tao (M'00-SM'04) received the B.S. degree from the PLA Electronic Engineering Institute, Hefei, China, in 1985, and the M.S. and Ph.D. degrees from the Harbin Institute of Technology, Harbin, China, in 1990 and 1993, respectively.

$\mathrm{He}$ was a Senior Visiting Scholar with the University of Michigan, Ann Arbor, MI, USA, and the University of Delaware, Newark, DE, USA, in 2001 and 2016, respectively. He is currently a Professor with the School of Information and Electronics, Beijing Institute of Technology, Beijing, China. He has three books and more than 100 peer-reviewed journal articles. His research interests include fractional Fourier transform and its applications, theory, and technology for radar and communication systems.

Dr. Tao is a fellow of the Institute of Engineering and Technology and the Chinese Institute of Electronics. He was a recipient of the National Science Foundation of China for Distinguished Young Scholars in 2006 and a Distinguished Professor of Changiiang Scholars Program in 2009. He has been a Chief Professor of the Creative Research Groups with the National Natural Science Foundation of China since 2014. He was a Chief Professor of the Program for Changjiang Scholars and Innovative Research Team in University from 2010 to 2012 . He is currently the Vice-Chair of the IEEE China Council. He is also the Vice-Chair of the International Union of Radio Science (URSI) China Council and a member of the Wireless Communication and Signal Processing Commission, URSI. He was a recipient of the First Prize of Science and Technology Progress in 2006 and 2007, and the First Prize of Natural Science in 2013, both awarded by the Ministry of Education. 